\title{
DISCRETE ANALOGUE OF GENERALIZED HARDY SPACES AND MULTIPLICATION OPERATORS ON HOMOGENOUS TREES
}

\author{
PERUMAL MUTHUKUMAR AND SAMINATHAN PONNUSAMY
}

\begin{abstract}
In this article, we define discrete analogue of generalized Hardy spaces and its separable subspace on a homogenous rooted tree and study some of its properties such as completeness, inclusion relations with other spaces, separability, growth estimate for functions in these spaces and their consequences. Equivalent conditions for multiplication operators to be bounded and compact are also obtained. Furthermore, we discuss about point spectrum, approximate point spectrum and spectrum of multiplication operators and discuss when a multiplication operator is an isometry.
\end{abstract}

\section{INTRODUCTION}

The theory of function spaces defined on the unit disk $\mathbb{D}=\{z \in \mathbb{C}:|z|<1\}$ is particulary a well developed subject. The recent book by Pavlović [20] and the book of Zhu 24] provide us with a solid foundation in studying various function spaces on the unit disk. One can also refer [15] for Hardy spaces $\left(H^{p}\right)$, [17] for Bergman spaces $\left(A^{p}\right)$, [16] for Dirichlet spaces $\left(\mathcal{D}_{p}\right)$ and [9] for Bloch space $(\mathcal{B})$.

In recent years, there has been a considerable interest in the study of function spaces on discrete set such as tree (more generally on graphs). For example, Lipschitz space of a tree (discrete analogue of Bloch space) [10], weighted Lipschitz space of a tree [4], iterated logarithmic Lipschitz space of a tree [3] and $H^{p}$ spaces on trees [18] are some in this line of investigation. In [18] the $H^{p}$ spaces on trees are defined by means of certain maximal or square function operators associated with a nearest neighbour transition operator which is very regular and this study was further developed in [6].

In the study of operators on function spaces, multiplication and composition operators arise naturally and play an important role. Moreover, study of composition operators on various function spaces on the unit disk is better developed than the multiplication operators, and the literature in these topics are exhaustive. See for example the survey articles [7, 19, 23] on multiplication operators on various function spaces of the unit disk.

In the case of operator theory on discrete function spaces, average operator (Laplacian operator) is studied more than other operators. Colonna and others studied

2000 Mathematics Subject Classification. Primary: 47B38, 05C05; Secondary: 46B50,46B26.

Key words and phrases. Rooted homogeneous tree, multiplication operators, Lipschitz space, generalized Hardy spaces

File: MPon1 ${ }^{\circ} 2016{ }^{*}$ final.tex, printed: 8-10-2018, 20.23. 
multiplication operators on Lipschitz space, weighted Lipschitz space, and iterated logarithmic Lipschitz space of a tree in [10], 4] and [3], respectively. In [2, 11], the authors discussed about multiplication operators between Lipschitz type spaces and the space of bounded functions on a tree. Recently, Allen et al. [5] have also studied composition operators on the Lipschitz space of a tree.

In this article, we define discrete analogue of generalized Hardy spaces and study its important properties. Also, we study multiplication operators on generalized Hardy spaces on homogeneous trees (i.e. every vertex has same number of neighbours).

\section{Preliminaries}

A graph $G$ is a pair $G=(V, E)$ of sets satisfying $E \subseteq V \times V$. The elements of $V$ and $E$ are called vertices and edges of the graph $G$, respectively. Two vertices $x, y \in V$ (with the abuse of language, one can write as $x, y \in G$ ) are said to be neighbours or adjacent (denoted by $x \sim y$ ) if there is an edge connecting them. A regular (homogeneous) graph is a graph in which every vertex has the same number of neighbours. If every vertex has $k$ neighbours, then the graph is said to be $k$-regular ( $k$-homogeneous) graph. A path is part of a graph with finite or infinite sequence of distinct vertices $\left[v_{0}, v_{1}, v_{2}, \ldots\right]$ such that $v_{n} \sim v_{n+1}$. If $P=\left[v_{0}-v_{1}-v_{2}-\cdots-v_{n}\right]$ is a path then the graph $C=\left[v_{0}-v_{1}-v_{2}-\cdots-v_{n}-v_{0}\right]$ (path $P$ with an additional edge $\left.v_{n} v_{0}\right)$ is called a cycle. A non-empty graph $G$ is called connected if any two of its vertices are linked by a path in $G$. A connected and locally finite (every vertex has finite number of neighbours) graph without cycles, is called a tree. A rooted tree is a tree in which a special vertex (called root) is singled out. The distance between any two vertex of a tree is the number of edges in the unique path connecting them. If $G$ is a rooted tree with root $o$, then $|v|$ denotes the distance between $o$ and $v$. Further the parent (denoted by $v^{-}$) of a vertex $v$ is the unique vertex $w \in G$ such that $w \sim v$ and $|w|=|v|-1$. For basic issues concerning graph theory, one can refer to standard texts such as [13].

Let $T$ be a rooted tree. By a function defined on a graph, we mean a function defined on its vertices. The Lipschitz space and the weighted Lipschitz space of $T$ are denoted by $\mathcal{L}$ and $\mathcal{L}_{w}$, respectively. These are defined as follows:

$$
\mathcal{L}=\left\{f: T \rightarrow \mathbb{C}: \sup _{v \in T, v \neq o}\left|f(v)-f\left(v^{-}\right)\right|<\infty\right\}
$$

and

$$
\mathcal{L}_{w}=\left\{f: T \rightarrow \mathbb{C}: \sup _{v \in T, v \neq o}|v|\left|f(v)-f\left(v^{-}\right)\right|<\infty\right\}
$$

respectively. Throughout the discussion, a homogeneous rooted tree with infinite vertices is denoted by $T, \mathbb{N}=\{1,2, \ldots\}$ and $\mathbb{N}_{0}=\mathbb{N} \cup\{0\}$. 
For $p \in(0, \infty]$, the generalized Hardy space $H_{g}^{p}(\mathbb{D})$ consists of all those measurable functions $f: \mathbb{D} \rightarrow \mathbb{C}$ such that $M_{p}(r, f)$ exists for all $r \in[0,1)$ and $\|f\|_{p}<\infty$, where

$$
\|f\|_{p}= \begin{cases}\sup _{0 \leq r<1} M_{p}(r, f) & \text { if } p \in(0, \infty), \\ \sup _{z \in \mathbb{D}}|f(z)| & \text { if } p=\infty\end{cases}
$$

and

$$
M_{p}^{p}(r, f)=\frac{1}{2 \pi} \int_{0}^{2 \pi}\left|f\left(r e^{i \theta}\right)\right|^{p} d \theta
$$

The classical Hardy space $H^{p}$ is a subspace of $H_{g}^{p}(\mathbb{D})$ consists of only analytic functions. See [8] for recent investigation on $H_{g}^{p}(\mathbb{D})$ and some related function spaces.

For our investigation this definition has an analog in the following form.

Definition 1. Let $T$ be a $q+1$ homogeneous tree rooted at $o$. For every $n \in \mathbb{N}$, we introduce

$$
M_{p}(n, f):= \begin{cases}\left(\frac{1}{(q+1) q^{n-1}} \sum_{|v|=n}|f(v)|^{p}\right)^{\frac{1}{p}} & \text { if } p \in(0, \infty) \\ \max _{|v|=n}|f(v)| & \text { if } p=\infty,\end{cases}
$$

$M_{p}(0, f):=|f(o)|$ and

$$
\|f\|_{p}:=\sup _{n \in \mathbb{N}_{0}} M_{p}(n, f) .
$$

The discrete analogue of the generalized Hardy space, denoted by $\mathbb{T}_{q, p}$, is then defined by

$$
\mathbb{T}_{q, p}:=\left\{f: T \rightarrow \mathbb{C} \text { such that }\|f\|_{p}<\infty\right\} .
$$

Similarly, the discrete analogue of the generalized little Hardy space, denoted by $\mathbb{T}_{q, p, 0}$, is defined by

$$
\mathbb{T}_{q, p, 0}:=\left\{f \in \mathbb{T}_{q, p}: \lim _{n \rightarrow \infty} M_{p}(n, f)=0\right\}
$$

for every $p \in(0, \infty]$. For the sake of simplicity, we shall write $\mathbb{T}_{q, p}$ and $\mathbb{T}_{q, p, 0}$ as $\mathbb{T}_{p}$ and $\mathbb{T}_{p, 0}$, respectively. Unless otherwise stated explicitly, throughout $\|\cdot\|_{p}$ is defined as above.

The paper is organized as follows. In several sub sections of Section 3, we investigate important properties such as completeness, inclusion relations and separability of the spaces $\mathbb{T}_{p}$ and $\mathbb{T}_{p, 0}$. First, we prove that $\mathbb{T}_{p}$ and $\mathbb{T}_{p, 0}$ are Banach spaces (see Theorems 1 and 2). As with the $H^{p}$ spaces, similar inclusion relations are derived for the discrete cases (see Theorem 3). Also, we show that $\mathbb{T}_{p}$ is not a separable space whereas $\mathbb{T}_{p, 0}$ is separable (see Theorems 4 and 5 ). Finally, in Section 3 , we compare the convergence in norm and uniform convergence on compact sets of $T$. In Section 4. we study multiplication operators on generalized Hardy spaces on homogeneous trees. In particular, we give characterization of bounded and compact multiplication operators on $\mathbb{T}_{p}$ (resp. on $\mathbb{T}_{p, 0}$ ), see Theorems 6 and 8 , Also we determine the point spectrum, the approximate point spectrum and the spectrum for the multiplication 
operator $M_{\psi}$ and determine an upper bound for the essential norm of $M_{\psi}$. Finally, we provide necessary and sufficient condition for a multiplication operator to be an isometry.

\section{Topological Properties of $\mathbb{T}_{p}$ AND $\mathbb{T}_{p, 0}$}

\subsection{Completeness.}

Theorem 1. For $1 \leq p \leq \infty,\|\cdot\|_{p}$ induces a Banach space structure on the space $\mathbb{T}_{p}$

Proof. First we begin with the case $p=\infty$. In this case, (2.1) reduces to $\|f\|_{p}=$ $\sup _{v \in T}|f(v)|$ and thus, the space $\mathbb{T}_{\infty}$ coincides with the set of all bounded functions on $T$ with sup-norm which is known to be a Banach space.

Next, we consider the case $1 \leq p<\infty$. We have the following.

(i) If $f \equiv 0$, then $\|f\|_{p}=0$. Conversely, if $\|f\|_{p}=0$ then $M_{p}(n, f)=0$ for all $n \in \mathbb{N}_{0}$ showing that $\sum_{|v|=n}|f(v)|^{p}=0$ for all $n \in \mathbb{N}_{0}$ and thus, $f \equiv 0$.

(ii) For each $n \in \mathbb{N}_{0}$ and $\alpha \in \mathbb{C}$, it is easy to see by definition that $M_{p}(n, \alpha f)=$ $|\alpha| M_{p}(n, f)$ and thus, $\|\alpha f\|_{p}=|\alpha|\|f\|_{p}$.

(iii) For each $n \in \mathbb{N}$ and $f, g \in \mathbb{T}_{p}$, one has (since $p \geq 1$ )

$$
\begin{aligned}
M_{p}(n, f+g) & =\left\{\frac{1}{(q+1) q^{n-1}} \sum_{|v|=n}(|f(v)|+|g(v)|)^{p}\right\}^{\frac{1}{p}} \\
& \leq\left(\frac{1}{(q+1) q^{n-1}} \sum_{|v|=n}|f(v)|^{p}\right)^{\frac{1}{p}}+\left(\frac{1}{(q+1) q^{n-1}} \sum_{|v|=n}|g(v)|^{p}\right)^{\frac{1}{p}} \\
& =M_{p}(n, f)+M_{p}(n, g) .
\end{aligned}
$$

The last inequality trivially holds for $n=0$ and thus, $\|f+g\|_{p} \leq\|f\|_{p}+\|g\|_{p}$. Hence $\left(\mathbb{T}_{p},\|\cdot\|_{p}\right)$ is a normed linear space. In order to prove that $\mathbb{T}_{p}$ is a Banach space, we begin with a Cauchy sequence $\left\{f_{k}\right\}$ in $\mathbb{T}_{p}$. Then $\left\{f_{k}(v)\right\}$ is a Cauchy sequence in $\mathbb{C}$ for every $v \in T$ and thus, $\left\{f_{k}\right\}$ converges pointwise to a function $f$. Now, for a given $\epsilon>0$, there exists an $N \in \mathbb{N}$ such that $M_{p}\left(n, f_{k}-f_{l}\right)<\epsilon$ for all $k, l \geq N$ and $n \in \mathbb{N}_{0}$. Letting $l \rightarrow \infty$, we get $M_{p}\left(n, f_{k}-f\right) \leq \epsilon$ for all $k \geq N$ and $n \in \mathbb{N}_{0}$. Hence $\left\|f_{k}-f\right\|_{p} \leq \epsilon$ for all $k \geq N$, which gives that $f_{k} \rightarrow f$. The triangle inequality $\|f\|_{p} \leq\left\|f-f_{N}\right\|_{p}+\left\|f_{N}\right\|_{p}$ gives that $f \in \mathbb{T}_{p}$. This completes the proof of the theorem.

Remark 1. For $0<p<1, \mathbb{T}_{p}$ is obviously a complete metric space.

A function $f: T \rightarrow \mathbb{C}$ is said to be radial constant function if $f(v)=f(w)$ whenever $|v|=|w|$. 
Remark 2. Since the integral means $M_{p}(r, f)$ of an analytic function $f$ defined on $\mathbb{D}$ is an increasing function of $r$, we have $\|f\|_{p}=\lim _{r \rightarrow 1^{-}} M_{p}(r, f)$. Thus, the little Hardy space $H_{0}^{p}$, defined by

$$
H_{0}^{p}:=\left\{f \in H^{p}: M_{p}(r, f) \rightarrow 0 \text { as } r \rightarrow 1^{-}\right\},
$$

consists of only a single element, namely, the zero function. But this is not the case in the generalized Hardy space $H_{g}^{p}(\mathbb{D})$ of measurable functions, This is because the maximum modulus principle is not valid for a general element in $H_{g}^{p}(\mathbb{D})$. Consequently, the generalized little Hardy space $H_{0, g}^{p}$ is non-trivial (i.e. not a zero subspace), where

$$
H_{0, g}^{p}:=\left\{f \in H_{g}^{p}(\mathbb{D}): M_{p}(r, f) \rightarrow 0 \text { as } r \rightarrow 1^{-}\right\} .
$$

For example, if

$$
f_{\alpha}(z)= \begin{cases}1 & \text { for }|z| \leq \alpha \\ 0 & \text { for } \alpha<|z|<1\end{cases}
$$

then $f \in H_{0, g}^{p}$ for each $0 \leq \alpha<1$.

In the discrete case, $\mathbb{T}_{p, 0}$ is non-trivial. In fact, the set of all radial constant functions in $\mathbb{T}_{p, 0}$ is isometrically isomorphic to the sequence space $c_{0}$ (set of all sequences that converges to zero).

Here are few questions that arise naturally.

Question 1. Is it possible to define an inner product so that $\mathbb{T}_{2}$ becomes a Hilbert space? As with the $l^{p}$ and the $H^{p}$ spaces, whether $\mathbb{T}_{p}$ is not isomorphic to $\mathbb{T}_{q}$ when $p \neq q$ ? What can be said about the dual of $\mathbb{T}_{p}$ ?

These questions are open for the moment.

Theorem 2. For $1 \leq p \leq \infty,\|\cdot\|_{p}$ induces a Banach space structure on $\mathbb{T}_{p, 0}$.

Proof. For $n \in \mathbb{N}_{0}$ and $f, g \in \mathbb{T}_{p}$, we easily have

$$
M_{p}(n, \alpha f)=|\alpha| M_{p}(n, f) \text { and } M_{p}(n, f+g) \leq M_{p}(n, f)+M_{p}(n, g)
$$

so that $\mathbb{T}_{p, 0}$ is a subspace of $\mathbb{T}_{p}$. Suppose $\left\{f_{k}\right\}$ is a Cauchy sequence in $\mathbb{T}_{p, 0}$. Since $\mathbb{T}_{p}$ is a Banach space, $\left\{f_{k}\right\}$ converges to some function $f \in \mathbb{T}_{p}$. Next, we need to prove that $f \in \mathbb{T}_{p, 0}$, i.e., $M_{p}(n, f) \rightarrow 0$ as $n \rightarrow \infty$. To do this, let $\epsilon>0$ be given. Then there exists a $k \in \mathbb{N}$ such that $\left\|f_{k}-f\right\|_{p}<\epsilon / 2$. Since $f_{k} \in \mathbb{T}_{p, 0}$, we can choose $N \in \mathbb{N}$ so that $M_{p}\left(n, f_{k}\right)<\epsilon / 2$ for all $n \geq N$. From the inequality $M_{p}(n, f) \leq M_{p}\left(n, f-f_{k}\right)+M_{p}\left(n, f_{k}\right)$, it follows that $M_{p}(n, f)<\epsilon$ for all $n \geq N$. Thus, $f \in \mathbb{T}_{p, 0}$ which completes the proof.

\subsection{Inclusion Relations.}

Lemma 1. For $0<r<s \leq \infty$ and for every complex valued function $f$ on $T$, $M_{r}(n, f) \leq M_{s}(n, f)$ holds for all $n \in \mathbb{N}_{0}$. 
Proof. The result for $s=\infty$ follows from the definition of $M_{r}(n, f)$ and thus, it suffices to prove the lemma for the case $0<r<s<\infty$. Again by Definition 1, we see that

$$
M_{r}(0, f)=|f(o)|=M_{s}(0, f) .
$$

For $n \in \mathbb{N}$, we have $(q+1) q^{n-1}$ vertices with $|v|=n$. Recall that on the Euclidean space $\mathbb{C}^{N}$, the following norm equivalence is well-known for $0<r<s$ :

$$
\|x\|_{s} \leq\|x\|_{r} \leq N^{\frac{1}{r}-\frac{1}{s}}\|x\|_{s}
$$

where $p$-norm $\|\cdot\|_{p}$ on $\mathbb{C}^{N}$ is given by $\|x\|_{p}^{p}=\sum_{k=1}^{N}\left|x_{k}\right|^{p}$. The second inequality in (3.1), is an easy consequence of Hölder's inequality for finite sum. We may now use this with $N=(q+1) q^{n-1}$. As a consequence of it, we have

$$
\left(\sum_{|v|=n}|f(v)|^{r}\right)^{\frac{1}{r}} \leq\left\{(q+1) q^{n-1}\right\}^{\frac{1}{r}-\frac{1}{s}}\left(\sum_{|v|=n}|f(v)|^{s}\right)^{\frac{1}{s}}
$$

which may be rewritten as

$$
\left(\frac{1}{(q+1) q^{n-1}} \sum_{|v|=n}|f(v)|^{r}\right)^{\frac{1}{r}} \leq\left(\frac{1}{(q+1) q^{n-1}} \sum_{|v|=n}|f(v)|^{s}\right)^{\frac{1}{s}} .
$$

This shows that $M_{r}(n, f) \leq M_{s}(n, f)$ for all $n \in \mathbb{N}_{0}$. The proof is complete.

As an immediate consequence of Lemma 1, one has the following.

Theorem 3. For $0<r<s \leq \infty$, we have $\mathbb{T}_{s} \subset \mathbb{T}_{r}$ and $\mathbb{T}_{s, 0} \subset \mathbb{T}_{r, 0}$.

We now show by an example that the inclusions in Theorem 3 is proper. Let $0<r<p<s \leq \infty$. Choose a sequence of vertices $\left\{v_{n}\right\}$ such that $\left|v_{n}\right|=n$ for all $n \in \mathbb{N}$. Consider the function $f$ defined by

$$
f(v)=\left\{\begin{array}{cl}
\left\{(q+1) q^{n-1}\right\}^{\frac{1}{p}} & \text { if } v=v_{n} \text { for some } n \in \mathbb{N} \\
0 & \text { elsewhere. }
\end{array}\right.
$$

Then, for $n \in \mathbb{N}$, one has

$$
M_{r}(n, f)=\left\{(q+1) q^{n-1}\right\}^{\frac{1}{p}-\frac{1}{r}}
$$

so that $M_{r}(n, f) \rightarrow 0$ as $n \rightarrow \infty$, since $p>r$. Also, we have

$$
M_{s}(n, f)= \begin{cases}\left\{(q+1) q^{n-1}\right\}^{\frac{1}{p}} & \text { if } s=\infty \\ \left\{(q+1) q^{n-1}\right\}^{\frac{1}{p}-\frac{1}{s}} & \text { if } s<\infty\end{cases}
$$

and in either case, we find that $M_{s}(n, f) \rightarrow \infty$ as $n \rightarrow \infty$. This example shows that $\mathbb{T}_{s, 0}$ is a proper subspace of $\mathbb{T}_{r, 0}$. From this example, it can be also seen that $\mathbb{T}_{s}$ is a proper subspace of $\mathbb{T}_{r}$. 
Remarks 1. (1) The unbounded function $f(v)=|v|$ belongs to $\mathcal{L}$ but is not in $\mathbb{T}_{p}$ for any $0<p<\infty$. On the other hand, let us now fix an infinite path $o-v_{1}-v_{2} \cdots$ with $\left|v_{k}\right|=k$. Define $g(v)=\left\{(q+1) q^{k-1}\right\}^{\frac{1}{p}}$ if $v=v_{k}$ and 0 otherwise. It is then easy to check that $g$ belongs to $\mathbb{T}_{p}$ for all $0<p<\infty$ but is not in $\mathcal{L}$. Thus, $\mathcal{L}$ is not comparable with $\mathbb{T}_{p}$ for all $0<p<\infty$.

(2) Consider the radial constant function $h$ defined by $h(o)=0$ and

$$
h(v)=\sum_{k=1}^{|v|} \frac{1}{k} \text { if }|v| \geq 1 .
$$

By simple calculations, we find that $h$ belongs to $\mathcal{L}_{w}$ but is not in $\mathbb{T}_{p}$ for any $0<p<\infty$. For the other direction, we fix an infinite path $o-v_{1}-v_{2} \cdots$ with $\left|v_{k}\right|=k$. If $A=\left\{o, v_{1}, v_{2}, \ldots\right\}$ then the characteristic function $\chi_{A}$, namely, $\chi_{A}(v)=1$ for $v \in A$ and zero elsewhere, belongs to $\mathbb{T}_{p}$ for all $0<p<\infty$ but is not in $\mathcal{L}_{w}$. This concludes the proof that $\mathcal{L}_{w}$ is not comparable with $\mathbb{T}_{p}$ for all $0<p<\infty$.

(3) Clearly, $\mathbb{T}_{\infty} \subseteq\left(\bigcap_{0<p<\infty} \mathbb{T}_{p}\right) \cap \mathcal{L}$, whereas $\mathcal{L}_{w}$ is not comparable with $\mathbb{T}_{\infty}$. For 2-homogeneous trees, this inclusion relation becomes an equality. This is because of the fact that there is no unbounded function in $\mathbb{T}_{p}$ for 2-homogeneous trees, which can be observed from the definition of $\mathbb{T}_{p}$.

3.3. Separability. In order to state results about the separability of $\mathbb{T}_{p, 0}$ and $\mathbb{T}_{p}$, we need to introduce few notations. Denote by $C_{c}(T)$ the set of all functions $f: T \rightarrow \mathbb{C}$ such that $M_{p}(n, f)=0$ for all but finitely many $n^{\prime}$ s. Also the closure of $C_{c}(T)$ under $\|\cdot\|_{p}$ is denoted by $\overline{C_{c}(T)}$.

Lemma 2. For $0<p \leq \infty$, we have $\overline{C_{c}(T)}=\mathbb{T}_{p, 0}$.

Proof. Let $f \in \mathbb{T}_{p, 0}$ and, for each $n$, define $\left\{f_{n}\right\}$ by

$$
f_{n}(v)=\left\{\begin{aligned}
f(v) & \text { if }|v| \leq n \\
0 & \text { otherwise }
\end{aligned}\right.
$$

Clearly, $f_{n} \in C_{c}(T)$ for each $n \in \mathbb{N}$ and

$$
M_{p}\left(k, f-f_{n}\right)=\left\{\begin{aligned}
M_{p}(k, f) & \text { if } k>n \\
0 & \text { otherwise. }
\end{aligned}\right.
$$

Therefore, we see that

$$
\left\|f-f_{n}\right\|_{p}=\sup _{m \in \mathbb{N}_{0}} M_{p}\left(m, f-f_{n}\right)=\sup _{m>n} M_{p}(m, f)
$$

and, because $M_{p}(m, f) \rightarrow 0$ as $m \rightarrow \infty$, it follows that $\left\|f-f_{n}\right\|_{p} \rightarrow 0$ as $n \rightarrow \infty$. This completes the proof.

Theorem 4. For $0<p \leq \infty, \mathbb{T}_{p, 0}$ is a separable space. 
Proof. It is easy to verify that $B=\left\{\chi_{\{v\}}: v \in T\right\}$ is a basis for $C_{c}(T)$. Since $B$ is countable, $C_{c}(T)$ is separable. Since $C_{c}(T)$ is dense in $\mathbb{T}_{p, 0}$, we conclude that $\mathbb{T}_{p, 0}$ is separable and the theorem follows.

We remark that $C_{c}(T)$ cannot be a Banach space with respect to any norm, since it has a countably infinite basis.

Theorem 5. For $0<p \leq \infty, \mathbb{T}_{p}$ is not separable.

Proof. Let $E \subset \mathbb{T}_{p}$ denote the set of all radial constant functions $f$ whose range is subset of $\{0,1\}$. Let $f, g \in E$ and $f \neq g$. Then there exists a $v \in T$ such that $f(v) \neq g(v)$. Since $f, g \in E$, we have $M_{p}(n, f-g) \leq 1$ for all $n$. On the other hand, $M_{p}(|v|, f-g)=1$ and hence, $\|f-g\|_{p}=\sup M_{p}(n, f-g)=1$. It is easy to check that $E$ is an uncountable subset of $\mathbb{T}_{p}$. Since any two distinct elements of $E$ must be of distance 1 apart and $E$ is uncountable, it follows that any dense subset of $\mathbb{T}_{p}$ cannot be countable. Consequently, $\mathbb{T}_{p}$ is not a separable space.

\subsection{Growth estimate and consequences.}

Lemma 3. Let $T$ be a $q+1$ homogeneous tree rooted at $o$ and $0<p<\infty$. Then, for $v \in T \backslash\{o\}$, we have the following:

(a) If $f \in \mathbb{T}_{p}$, then $|f(v)| \leq\left\{(q+1) q^{|v|-1}\right\}^{\frac{1}{p}}\|f\|_{p}$.

(b) If $f \in \mathbb{T}_{p, 0}$, then

The results are sharp.

$$
\lim _{|v| \rightarrow \infty} \frac{f(v)}{\left\{(q+1) q^{|v|-1}\right\}^{\frac{1}{p}}}=0 .
$$

Proof. Fix $v \in T \backslash\{o\}$ and let $n=|v|$. Then,

$$
|f(v)|^{p} \leq \sum_{|w|=n}|f(w)|^{p}=(q+1) q^{n-1} M_{p}^{p}(n, f)
$$

so that $|f(v)| \leq\left\{(q+1) q^{n-1}\right\}^{\frac{1}{p}} M_{p}(n, f)$ and thus,

$$
\frac{|f(v)|}{\left\{(q+1) q^{n-1}\right\}^{\frac{1}{p}}} \leq M_{p}(n, f) \leq\|f\|_{p} .
$$

The desired results follow.

In order to prove the sharpness, we fix $v \in T \backslash\{o\}$. Define $f(v)=\left\{(q+1) q^{|v|-1}\right\}^{\frac{1}{p}}$ and 0 elsewhere. We now let $m=|v|$ so that $M_{p}(n, f)=0$ for every $n \neq m$ and

$$
M_{p}(m, f)=\left(\frac{1}{(q+1) q^{m-1}} \sum_{|w|=m}|f(w)|^{p}\right)^{\frac{1}{p}}=\left(\frac{1}{(q+1) q^{m-1}}|f(v)|^{p}\right)^{\frac{1}{p}}=1 .
$$

We obtain that $\|f\|_{p}=\sup _{n \in \mathbb{N}_{0}} M_{p}(n, f)=1$ and hence,

$$
|f(v)|=f(v)=\left\{(q+1) q^{|v|-1}\right\}^{\frac{1}{p}}\|f\|_{p} .
$$


We conclude the proof.

Lemma 3(a) clearly holds if $v=o$, because $|f(o)| \leq\|f\|_{p}$.

Proposition 1. (Compare with [22, Corollary, p. 10]) Convergence in $\|\cdot\|_{p}(0<p \leq$ $\infty)$ implies uniform convergence on compact subsets of $T$.

Proof. The edge counting distance on $T$ induces the discrete metric. So, finite subsets are the only compact sets in $T$. Let $K$ be an arbitrary compact subset of $T$. Then there exists an $N \in \mathbb{N}$ such that $|v| \leq N$ for every $v \in K$. The proposition trivially holds for the case $p=\infty$, because given a function $f$ and a sequence $\left\{f_{n}\right\}$ converging to $f$ in norm,

$$
\sup _{v \in K}\left|\left(f_{n}-f\right)(v)\right| \leq\left\|f_{n}-f\right\|_{\infty} .
$$

Next, we consider the case $0<p<\infty$. From Lemma 3, given a function $f$ in $\mathbb{T}_{p}$, we have

$$
|f(v)| \leq\left\{(q+1) q^{|v|-1}\right\}^{\frac{1}{p}}\|f\|_{p} \leq\left\{(q+1) q^{N-1}\right\}^{\frac{1}{p}}\|f\|_{p} \text { for every } v \in K .
$$

This gives

$$
\sup _{v \in K}|f(v)| \leq\left\{(q+1) q^{N-1}\right\}^{\frac{1}{p}}\|f\|_{p}
$$

and thus, by replacing $f$ by $f_{n}-f$, we conclude that convergence in $\|\cdot\|_{p}$ implies uniform convergence on compact subsets of $T$.

Uniform convergence on compact subsets of $T$ does not necessarily imply the convergence in $\|\cdot\|_{p}(0<p \leq \infty)$ as can be seen from the following example.

Example 1. Consider the function $f \equiv 1$. For each $n$, define $\left\{f_{n}\right\}$ by

$$
f_{n}(v):=\left\{\begin{aligned}
f(v)(=1) & \text { if }|v| \leq n \\
0 & \text { otherwise. }
\end{aligned}\right.
$$

Then

$$
M_{p}\left(k, f-f_{n}\right)=\left\{\begin{array}{rc}
M_{p}(k, f)(=1) & \text { if } k>n \\
0 & \text { otherwise. }
\end{array}\right.
$$

Let $K$ be a compact subset of $T$. Then there exists an $N \in \mathbb{N}$ such that $|v| \leq N$ for every $v \in K$ and $\sup _{v \in K}\left|\left(f_{n}-f\right)(v)\right|=0$ for every $n>N$. It follows that $\left\{f_{n}\right\}$ converges uniformly on compact subsets of $T$ to $f$. On the other hand,

$$
\left\|f-f_{n}\right\|_{p}=\sup _{m \in \mathbb{N}_{0}} M_{p}\left(m, f-f_{n}\right)=1 \text { for every } n \in \mathbb{N} .
$$

Hence, $\left\{f_{n}\right\}$ does not converge to $f$ in $\|\cdot\|_{p}$.

From Proposition 1 and the above remark, we observe that the the topology of uniform convergence on the compact subsets of $T$ on $\mathbb{T}_{p}$ is similar to that of analytic cases such as $H^{p}$ spaces. This observation raises a natural question. Is $\mathbb{T}_{p}$ complete 
in the topology of uniform convergence on compact sets? The following example shows that the answer is negative. For each $n$, define $\left\{f_{n}\right\}$ by

$$
f_{n}(v):=\left\{\begin{aligned}
|v| & \text { if }|v| \leq n \\
0 & \text { otherwise. }
\end{aligned}\right.
$$

Let $K$ be a compact subset of $T$. Then there exists an $N \in \mathbb{N}$ such that $|v| \leq N$ for every $v \in K$. For $N<n<m, f_{n}(v)=f_{m}(v)$ for all $v \in K$. It is easy to see that $\left\{f_{n}\right\}$ is a Cauchy sequence in the topology of uniform convergence on compact sets and $\left\{f_{n}\right\}$ converges pointwise to the function $f(v)=|v|$. Note that $f$ can be the only possible limit of $\left\{f_{n}\right\}$ in the topology of uniform convergence on compact sets. Since $\mathbb{T}_{p}$ contains the sequence $\left\{f_{n}\right\}$ but not $f, \mathbb{T}_{p}$ cannot be complete under the topology of uniform convergence on compact sets.

\section{Multiplication Operators On $\mathbb{T}_{p}$ And $\mathbb{T}_{p, 0}$}

We now recall the following definitions.

Let $X$ be a complex normed linear space consisting of complex valued functions defined on a set $\Omega$. If $\psi$ is a complex valued function defined on $\Omega$, then the multiplication operator with symbol $\psi$ is defined by $M_{\psi} f=\psi f$ for every $f \in X$.

A Banach space $X$ on $\Omega$ said to be a functional Banach space if for each $v \in \Omega$, the point evaluation map $e_{v}: f \in X \mapsto f(v)$ is a bounded linear functional on $X$. The following result is well-known.

Lemma 4. [14, Lemma 11] Let $X$ be a functional Banach space on the set $\Omega$ and $\psi$ be a complex valued function on $\Omega$ such that $M_{\psi}$ maps $X$ into itself. Then $M_{\psi}$ is bounded on $X$ and $|\psi(v)| \leq\left\|M_{\psi}\right\|$ for all $v \in \Omega$. In particular, $\psi$ is bounded function.

It is natural to ask whether $\mathbb{T}_{p}$ and $\mathbb{T}_{p, 0}$ are functional Banach spaces.

\subsection{Boundedness.}

Proposition 2. For $1 \leq p \leq \infty, \mathbb{T}_{p}$ and $\mathbb{T}_{p, 0}$ are functional Banach spaces.

Proof. First we consider the case when $p=\infty$. Since $\left|e_{v}(f)\right|=|f(v)| \leq\|f\|_{\infty}$ for every $v \in T$, it follows that $\mathbb{T}_{\infty}$ is a functional Banach space.

Let us now consider the case when $1 \leq p<\infty$. The point evaluation map $e_{o}$ is a bounded linear functional on $\mathbb{T}_{p}$ because of the fact that $|f(o)| \leq\|f\|_{p}$ for every $f$ in $\mathbb{T}_{p}$. Now, we fix $v \in T$ and $v \neq o$. Then, from Lemma 3 , we have

$$
\left|e_{v}(f)\right|=|f(v)| \leq\left\{(q+1) q^{|v|-1}\right\}^{\frac{1}{p}}\|f\|_{p} \text { for every } f \in \mathbb{T}_{p} .
$$

So $e_{v}$ is a bounded linear functional on $\mathbb{T}_{p}$ with $\left\|e_{v}\right\| \leq\left\{(q+1) q^{|v|-1}\right\}^{\frac{1}{p}}$. Hence $\mathbb{T}_{p}$ is a functional Banach space. A similar proof works also for the space $\mathbb{T}_{p, 0}$. The proof is complete.

Theorem 6. Let $T$ be a $q+1$ homogeneous tree rooted at $o, \psi$ be a function on $T$. Let $X$ be $\mathbb{T}_{p}$ or $\mathbb{T}_{p, 0}$ or $C_{c}(T)$ with norm $\|\cdot\|_{p}$, where $0<p \leq \infty$. Then the following are equivalent (compare with [23, Proposition 2]). 
(a) $M_{\psi}$ is a bounded linear operator from $X$ to $X$,

(b) $\psi$ is a bounded function on $T$, i.e., $\psi \in \mathbb{T}_{\infty}$.

Proof. Let $X$ be $\mathbb{T}_{p}$ or $\mathbb{T}_{p, 0}$ or $C_{c}(T)$ with norm $\|\cdot\|_{p}$, where $0<p \leq \infty$.

(a) $\Rightarrow(\mathrm{b})$ : We will prove this implication by contradiction. Suppose that $\psi$ is an unbounded function on $T$. Then there exists a sequence of vertices $\left\{v_{k}\right\}$ such that

$$
\left|v_{1}\right|<\left|v_{2}\right|<\left|v_{3}\right| \cdots \text { and }\left|\psi\left(v_{k}\right)\right| \geq k \text {. }
$$

For each $k$, define $f_{k}: T \rightarrow \mathbb{C}$ by $f_{k}=C_{k, p} \chi_{\left\{v_{k}\right\}}$, where the constants $C_{k, p}$ 's are chosen in such a way that $\left\|f_{k}\right\|_{p}=1$. Note that $f_{k} \in X$ for each $k \in \mathbb{N}$. We obtain that

$$
k=k\left\|f_{k}\right\|_{p} \leq\left|\psi\left(v_{k}\right)\right|\left\|f_{k}\right\|_{p}=M_{p}\left(\left|v_{k}\right|, \psi f_{k}\right) \leq\left\|\psi f_{k}\right\|_{p}=\left\|M_{\psi} f_{k}\right\|_{p}
$$

for every $k \in \mathbb{N}$, which gives a contradiction to our assumption. Hence, $\psi$ is a bounded function on $T$.

(b) $\Rightarrow($ a) : Suppose that $\psi$ is a bounded function on $T$ and $0<p<\infty$. Then for any $f \in X$,

$$
\begin{aligned}
M_{p}\left(n, M_{\psi} f\right) & =\left(\frac{1}{(q+1) q^{n-1}} \sum_{|v|=n}\left|\left(M_{\psi} f\right)(v)\right|^{p}\right)^{\frac{1}{p}} \\
& =\left(\frac{1}{(q+1) q^{n-1}} \sum_{|v|=n}|\psi(v)|^{p}|f(v)|^{p}\right)^{\frac{1}{p}},
\end{aligned}
$$

which shows that

$$
M_{p}\left(n, M_{\psi} f\right) \leq\|\psi\|_{\infty} M_{p}(n, f) .
$$

For $p=\infty$, the inequality (4.1) is trivially holds. From (4.1), one can also observe that $M_{\psi} f \in X$ whenever $f \in X$. Taking the supremum over $n \in \mathbb{N}_{0}$ on both sides of (4.1), we deduce that $\left\|M_{\psi} f\right\|_{p} \leq\|\psi\|_{\infty}\|f\|_{p}$ and thus, $M_{\psi}$ is bounded linear operator from $X$ to $X$ with $\left\|M_{\psi}\right\| \leq\|\psi\|_{\infty}$.

Remark 3. Let $X$ be $\mathbb{T}_{p}$ or $\mathbb{T}_{p, 0}$ with norm $\|\cdot\|_{p}$, where $1 \leq p \leq \infty$. In this case, $X$ becomes a functional Banach space. Then by Lemma 4, one has $|\psi(v)| \leq\left\|M_{\psi}\right\|$ for all $v \in T$ which by taking the supremum gives $\|\psi\|_{\infty} \leq\left\|M_{\psi}\right\|$. Therefore, by Theorem [6, it follows that $\left\|M_{\psi}\right\|=\|\psi\|_{\infty}$.

4.2. Spectrum. Let $X$ be a normed linear space and $A$ be a bounded linear operator on $X$. The point spectrum $\sigma_{e}(A)$ of $A$ consists of all $\lambda \in \mathbb{C}$ such that $A-\lambda I$ is not injective. Thus $\lambda \in \sigma_{e}(A)$ if and only if there is some nonzero $x \in X$ such that $A(x)=\lambda x$. The approximate point spectrum $\sigma_{a}(A)$ of $A$ consists of all $\lambda \in \mathbb{C}$ such that $A-\lambda I$ is not bounded below. Thus $\lambda \in \sigma_{a}(A)$ if and only if there is a sequence $\left\{x_{n}\right\}$ in $X$ such that $\left\|x_{n}\right\|=1$ for each $n$ and $\left\|A\left(x_{n}\right)-\lambda x_{n}\right\| \rightarrow 0$ as $n \rightarrow \infty$. The spectrum $\sigma(A)$ of $A$ consists of all $\lambda \in \mathbb{C}$ such that $A-\lambda I$ is not invertible. 
It is clear from the definition that

$$
\sigma_{e}(A) \subseteq \sigma_{a}(A) \subseteq \sigma(A) .
$$

It is well-known that the spectrum of a bounded linear operator $A$ on a Banach space $X$ over $\mathbb{C}$ is a nonempty compact subset of $\mathbb{C}$ (see [12, Theorem 3.6]). Also, the boundary of the spectrum is contained in the approximate point spectrum of $A$ ([12, Proposition 6.7]) and approximate point spectrum is a closed subset of $\mathbb{C}$.

Theorem 7. For $1 \leq p \leq \infty$, let $X$ equal either $\mathbb{T}_{p}$ or $\mathbb{T}_{p, 0}$ or $C_{c}(T)$, and let $M_{\psi}$ be a bounded multiplication operator on $X$ with norm $\|.\|_{p}$. Then

(a) $\sigma_{e}\left(M_{\psi}\right)=$ Range of $\psi=\psi(T)$;

(b) $\sigma\left(M_{\psi}\right)=\sigma_{a}\left(M_{\psi}\right)=\overline{\psi(T)}$.

Proof. In order to prove (a), we begin by letting $\lambda \in \sigma_{e}\left(M_{\psi}\right)$. Then there exists a nonzero function $f \in X$ such that $\psi f=M_{\psi} f=\lambda f$. Since $f \neq 0$, there is a vertex $v$ such that $f(v) \neq 0$ and $(\psi(v)-\lambda) f(v)=0$. Thus, $\lambda=\psi(v) \in \psi(T)$ and therefore, $\sigma_{e}\left(M_{\psi}\right) \subseteq \psi(T)$.

Conversely, suppose that $\alpha \in \psi(T)$. Then, there exists a vertex $v$ such that $\psi(v)=\alpha$. Thus, $M_{\psi}\left(\chi_{\{v\}}\right)=\alpha \chi_{\{v\}}$ and $0 \neq \chi_{\{v\}} \in X$. This gives $\alpha \in \sigma_{e}\left(M_{\psi}\right)$. Hence, $\sigma_{e}\left(M_{\psi}\right)=\psi(T)$.

Before proving (b), we observe that for every $\lambda \in \mathbb{C}, M_{\psi}-\lambda I=M_{\psi-\lambda}$. Thus, $\lambda \in \sigma\left(M_{\psi}\right)$ if and only if $M_{\psi-\lambda}$ is not invertible if and only if $\frac{1}{\psi-\lambda}$ is not a bounded function on $T$ (see Theorem [6). Now, we let $\lambda \notin \overline{\psi(T)}$. Since the complement of $\overline{\psi(T)}$ is open, there exists an $r>0$ such that disk of radius $r$ centered at $\lambda$ is a subset of $\mathbb{C} \backslash \overline{\psi(T)}$. So, $|\psi(v)-\lambda| \geq r$ for every $v \in T$. Thus $\frac{1}{\psi-\lambda}$ is a bounded function and therefore, by Theorem [6, $M_{\frac{1}{\psi-\lambda}}$ is a bounded operator on $X$. It is easy to verify that $M_{\frac{1}{\psi-\lambda}}$ is the inverse of $M_{\psi-\lambda}$ and hence, $M_{\psi-\lambda}$ is invertible. We conclude that $\lambda$ cannot be in the spectrum, which in turn implies that $\sigma\left(M_{\psi}\right) \subseteq \overline{\psi(T)}$.

On the other hand, $\psi(T)=\sigma_{e}\left(M_{\psi}\right) \subseteq \sigma_{a}\left(M_{\psi}\right) \subseteq \sigma\left(M_{\psi}\right) \subseteq \overline{\psi(T)}$ and the fact that the approximate point spectrum and spectrum are closed subsets of $\mathbb{C}$ give that $\sigma\left(M_{\psi}\right)=\sigma_{a}\left(M_{\psi}\right)=\overline{\psi(T)}$.

Remark 4. Let $X$ be $\mathbb{T}_{p}$ or $\mathbb{T}_{p, 0}$ or $C_{c}(T)$ with $\|\cdot\|_{p}$, where $1 \leq p \leq \infty$. Then $M_{\psi}: X \rightarrow X$ is not injective if and only if $0 \in \sigma_{e}\left(M_{\psi}\right)=\psi(T)$. So, 0 is in the range of $\psi$ is a necessary and sufficient condition for $M_{\psi}$ not being injective on $X$.

\subsection{Compactness.}

Theorem 8. Let $X$ be either $\mathbb{T}_{p}$ or $\mathbb{T}_{p, 0}$, where $1 \leq p \leq \infty$ and let $M_{\psi}: X \rightarrow X$ be a bounded multiplication operator on $X$. Then $M_{\psi}$ is a compact operator on $X$ if and only if $\psi(v) \rightarrow 0$ as $|v| \rightarrow \infty$.

Proof. Let $M_{\psi}$ be a compact operator on $X$. Then, from [21, Theorem 4.25], $\sigma_{e}\left(M_{\psi}\right)=\psi(T)$ (as well as $\sigma\left(M_{\psi}\right)$ ) is a countable set with 0 as the only possible limit point. Suppose $\psi(v) \not \rightarrow 0$ as $|v| \rightarrow \infty$. Then there exists an $\epsilon>0$ and a 
sequence $\left\{v_{k}\right\}$ in $T$ such that $\left|v_{k}\right| \rightarrow \infty$ and $\left|\psi\left(v_{k}\right)\right| \geq \epsilon$ for all $k$. By BolzanoWeierstrass theorem, $\left\{\psi\left(v_{k}\right)\right\}$ has a limit point. Because $\left|\psi\left(v_{k}\right)\right| \geq \epsilon$ for all $k$, we have a contradiction to the fact that 0 is the only possible limit point. Hence, $\psi(v) \rightarrow 0$ as $|v| \rightarrow \infty$.

For the proof of the converse part, we use the fact that the set of compact operators on $X$ is a closed subspace of the set of all bounded operators on $X$ (see [21, Theorem 4.18 part (c)]).

Consider a function $\psi$ from $C_{c}(T)$. Then there exists an $N \in \mathbb{N}$ such that $\psi(v)=0$ for every $|v|>N$. So, $\left(M_{\psi} f\right)(v)=\psi(v) f(v)=0$ for every $|v|>N$ and for every $f \in X$. Thus, the range of $M_{\psi}$ is a finite dimensional subspace, which shows that $M_{\psi}$ is a compact operator ([21, Theorem 4.18 part (a)]).

Let $\psi$ be a arbitrary function such that $\psi(v) \rightarrow 0$ as $|v| \rightarrow \infty$. For each $n$, define $\left\{f_{n}\right\}$ by

$$
f_{n}(v):=\left\{\begin{aligned}
\psi(v) & \text { if }|v| \leq n \\
0 & \text { otherwise. }
\end{aligned}\right.
$$

By definition $f_{n} \in C_{c}(T)$ for every $n$. Thus, $M_{f_{n}}$ is a compact operator for every $n$. Moreover,

$$
\left\|M_{f_{n}}-M_{\psi}\right\|=\left\|M_{f_{n}-\psi}\right\|=\left\|f_{n}-\psi\right\|_{\infty}=\sup _{|v|>n}|\psi(v)|,
$$

which approaches to zero as $|v| \rightarrow \infty$, because $\psi(v) \rightarrow 0$ as $|v| \rightarrow \infty$. Thus, $M_{\psi}$ is the limit (in the operator norm) of a sequence $\left\{M_{f_{n}}\right\}$ of compact operators, and hence, $M_{\psi}$ is compact on $X$. The proof is now complete.

Lemma 5. Let $X$ be either $\mathbb{T}_{p}$ or $\mathbb{T}_{p, 0}$, where $1 \leq p \leq \infty$ and let $M_{\psi}: X \rightarrow X$ be a bounded multiplication operator on $X$. If $M_{\psi}$ is a compact operator on $X$, then, for every bounded sequence $\left\{f_{n}\right\}$ in $X$ converging to 0 pointwise, the sequence $\left\|\psi f_{n}\right\| \rightarrow 0$ as $n \rightarrow \infty$.

Proof. Suppose that $\left\{g_{n}\right\}$ in $X$ is a bounded sequence converging to 0 pointwise. Since $M_{\psi}$ is a compact operator, there is a subsequence $\left\{g_{n_{k}}\right\}$ of $\left\{g_{n}\right\}$ such that $\left\{\psi g_{n_{k}}\right\}=\left\{M_{\psi}\left(g_{n_{k}}\right)\right\}$ converges in $\|.\|_{p}$ to some function, say, $g$. It follows that $\left\{\psi g_{n_{k}}\right\}$ converges to $g$ pointwise. Since the convergence of $\left\{g_{n}\right\}$ to 0 implies that $g \equiv 0$, we deduce that $\left\{\psi g_{n_{k}}\right\}$ converges to 0 in $\|\cdot\|_{p}$.

Let $\left\{f_{n}\right\}$ be a bounded sequence in $X$ converging to 0 pointwise. We claim that $\left\|M_{\psi}\left(f_{n}\right)\right\|=\left\|\psi f_{n}\right\| \rightarrow 0$ as $n \rightarrow \infty$. Suppose that $\left\|M_{\psi}\left(f_{n}\right)\right\| \not \rightarrow 0$ as $n \rightarrow \infty$. Then there exists a subsequence $\left\{f_{n_{j}}\right\}$ and an $\epsilon>0$ such that $\left\|M_{\psi}\left(f_{n_{j}}\right)\right\| \geq \epsilon$ for all $j$. By taking $g_{n}=f_{n_{j}}$ in the last paragraph, we find that $\left\{\psi g_{n_{k}}\right\}$ converges to 0 in $\|\cdot\|_{p}$, which is not possible because $\left\|M_{\psi}\left(f_{n_{j}}\right)\right\| \geq \epsilon$ for all $j$. Hence, $\left\|M_{\psi}\left(f_{n}\right)\right\|=\left\|\psi f_{n}\right\| \rightarrow$ 0 as $n \rightarrow \infty$, and the proof is complete.

It is well-known (cf. [12, Proposition 3.3]) that if $A$ is a compact operator on a normed linear space $Y$, then the image of every weak convergent sequence is norm convergent, i.e., $x_{n} \rightarrow x$ weakly implies that $A x_{n} \rightarrow A x$. In view of this result and Lemma 5, we have a natural question: Is there a relationship between weak 
convergence to 0 in $X$ and the boundedness of a sequence in $X$ converging to 0 pointwise?

4.4. Upper bound for the essential norm. Let $C L(X)$ denote the set of all compact operators on $X$. The essential norm $\|A\|_{e}$ of an bounded operator $A$ on $X$ defined to be the distance between $A$ and $C L(X)$ :

$$
\|A\|_{e}=\operatorname{dist}(A, C L(X))=\inf \{\|A-K\|: K \in C L(X)\} .
$$

The following theorem is a natural generalization of Theorem 8 ,

Theorem 9. Let $M_{\psi}$ be a bounded multiplication operator on $\mathbb{T}_{p}$ or on $\mathbb{T}_{p, 0}$, where $1 \leq p \leq \infty$. Then

$$
\left\|M_{\psi}\right\|_{e} \leq \limsup _{n \rightarrow \infty} M_{\infty}(n, \psi)=\lim _{n \rightarrow \infty} \sup _{|v| \geq n}|\psi(v)| .
$$

Proof. For each $n \in \mathbb{N}$, define $\left\{\psi_{n}\right\}$ by

$$
\psi_{n}(v):=\left\{\begin{aligned}
\psi(v) & \text { if }|v|<n \\
0 & \text { otherwise. }
\end{aligned}\right.
$$

Clearly, $M_{\psi_{n}}$ is a compact operator for every $n \in \mathbb{N}$ and thus, for every $n \in \mathbb{N}$,

$$
\begin{aligned}
\left\|M_{\psi}\right\|_{e} & =\inf \left\{\left\|M_{\psi}-K\right\|: K \in C L(X)\right\} \\
& \leq\left\|M_{\psi}-M_{\psi_{n}}\right\|=\left\|\psi-\psi_{n}\right\|_{\infty} \\
& =\sup _{|v| \geq n}|\psi(v)|=\sup _{m \geq n} M_{\infty}(m, \psi) .
\end{aligned}
$$

Hence, $\left\|M_{\psi}\right\|_{e} \leq \inf _{n \in \mathbb{N}} \sup _{|v| \geq n}|\psi(v)|=\limsup _{n \rightarrow \infty} M_{\infty}(n, \psi)$ and the proof is complete.

We would like to point out that one way implication of Theorem 8 follows from Theorem 9. Indeed if if $\psi(v) \rightarrow 0$ as $|v| \rightarrow \infty$, then Theorem 9 gives that $\left\|M_{\psi}\right\|_{e}=0$ and thus, $M_{\psi}$ is a compact operator.

4.5. Isometry. A map $A$ from a normed linear space $\left(X,\|\cdot\|_{X}\right)$ to a normed linear space $\left(Y,\|\cdot\|_{Y}\right)$ is said to be an isometry if $\|A x\|_{Y}=\|x\|_{X}$ for all $x \in X$.

Theorem 10. Let $X$ be either $\mathbb{T}_{p}$ or $\mathbb{T}_{p, 0}$, where $0<p \leq \infty$ and let $M_{\psi}: X \rightarrow X$ be a bounded multiplication operator on $X$. Then $M_{\psi}$ is an isometry on $X$ if and only if $|\psi(v)|=1$ for all $v \in T$.

Proof. Suppose that $|\psi(v)|=1$ for all $v \in T$. Then $M_{p}(n, \psi f)=M_{p}(n, f)$ for all $n$, which shows that $\|f\|_{p}=\|\psi f\|_{p}=\left\|M_{\psi}(f)\right\|_{p}$ and thus, $M_{\psi}$ is an isometry on $X$.

Conversely, suppose that $M_{\psi}$ is an isometry on $X$. First we consider the case $p=\infty$. Let $f$ be $\chi_{\{v\}}$. Because $M_{\psi}$ is an isometry on $X$, we have

$$
|\psi(v)|=\left\|M_{\psi}(f)\right\|_{\infty}=\|\psi f\|_{\infty}=\|f\|_{\infty}=1,
$$

which holds for every $v \in T$. Hence $|\psi(v)|=1$ for all $v \in T$. 
Next, we consider the case $0<p<\infty$. Let $f$ be $\chi_{\{o\}}$. Since $M_{\psi}$ is an isometry, $|\psi(o)|=1$. Take an arbitrary element $v \in T$ with $|v| \geq 1$ and let $f$ be $\chi_{\{v\}}$. Moreover, since $M_{\psi}$ is an isometry, we have

$$
\left(\frac{|\psi(v)|^{p}}{(q+1) q^{n-1}}\right)^{\frac{1}{p}}=\left\|M_{\psi}(f)\right\|_{p}=\|f\|_{p}=\left(\frac{1}{(q+1) q^{n-1}}\right)^{\frac{1}{p}},
$$

which shows that $|\psi(v)|=1$ for all $v \in T$.

Acknowledgement. The authors thank the referee for many useful comments which improve the presentation considerably. The first author thanks the Council of Scientific and Industrial Research (CSIR), India, for providing financial support in the form of a SPM Fellowship to carry out this research. The second author is on leave from the Indian Institute of Technology Madras, India.

\section{REFERENCES}

1. A. Aleman, P. L. Duren, M. J. Martin and D. Vukotić, Multiplicative isometries and isometric zero-divisors, Canad. J. Math. 62(2010), 961-974.

2. R. F. Allen, F. Colonna and G. R. Easley, Multiplication operators between Lipschitz-type spaces on a tree, Int. J. Math. Math. Sci. 2011, Art. ID 472495, 36 pp.

3. R. F. Allen, F. Colonna and G. R. Easley, Multiplication operators on the iterated logarithmic Lipschitz spaces of a tree, Mediterr. J. Math. 9(2012), 575-600.

4. R. F. Allen, F. Colonna and G. R. Easley, Multiplication operators on the weighted Lipschitz space of a tree, J. Operator Theory 69(2013), 209-231.

5. R. F. Allen, F. Colonna and G. R. Easley, Composition operators on the Lipschitz space of a tree, Mediterr. J. Math. 11(2014), 97-108.

6. F. D. Biase and M. A. Picardello, The Green formula and $H^{p}$ spaces on trees, Math. Z. 218(1995), 253-272.

7. D. M. Campbell and R. J. Leach, A survey of $H^{p}$ multipliers as related to classical function theory, Complex Variables Theory Appl. 3(1984), 85-111.

8. Sh. Chen, S. Ponnusamy and A. Rasila, On characterizations of Bloch-type, Hardy-type and Lipschitz-type spaces, Math. Z. 279(2015), 163-183.

9. J. G. Clunie, J. M. Anderson and Ch. Pommerenke, On Bloch functions and normal functions, J. Reine Angew. Math. 270(1974), 12-37.

10. F. Colonna and G. R. Easley, Multiplication operators on the Lipschitz space of a tree, Integr. Equ. Oper. Theory 68(2010), 391-411.

11. F. Colonna and G. R. Easley, Multiplication operators between the Lipschitz space and the space of bounded functions on a tree, Mediterr. J. Math. 9(2012), 423-438.

12. J. B. Conway, A course in functional analysis, 2nd edition, Springer, New York, 1990.

13. R. Diestel, Graph Theory, 4th edition, Springer, New York, 2010.

14. P. L. Duren, B. W. Romberg, and A. L. Shields, Linear functionals on $H^{p}$ spaces with $0<$ $p<1$, J. Reine Angew. Math. 238(1969), 32-60.

15. P. L. Duren, Theory of $H^{p}$ Spaces, Academic. Press, New York and London, 1970.

16. O. El-Fallah, K. Kellay, J. Mashreghi and T. Ransford, A primer on the Dirichlet space, Cambridge University Press, Cambridge, 2014.

17. H. Hedenmalm, B. Korenblum and K. Zhu, Theory of Bergman spaces, Springer-Verlag, New York 2000.

18. A. Korányi, M. A. Picardello and M. H. Taibleson, Hardy spaces on non-homogeneous trees, Symp. Math. 29(1987), 205-265. 
19. J. S. Manhas, Composition operators and multiplication operators on weighted spaces of analytic functions, Int. J. Math. Math. Sci. 2007, Art. ID 92070, 21 pp.

20. M. Pavlović, Function classes on the unit disc. An introduction, De Gruyter Studies in Mathematics, 52. De Gruyter, Berlin, 2014.

21. W. Rudin, Functional analysis, 2nd edition, International Series in Pure and Applied Mathematics, McGraw-Hill, Inc., New York, 1991.

22. J. H. Shapiro, Composition operators and classical function theory, Springer, New York, 1993.

23. D.Vukotić, Analytic Toeplitz operators on the Hardy space $H^{p}$ : a survey, Bull. Belg. Math. Soc. 10(2003), 101-113.

24. K. Zhu, Operator theory in function spaces, Marcel Dekker, New York, 1990.

P. Muthukumar, Indian Statistical Institute (ISI), Chennai Centre, SETS (Society for Electronic Transactions and Security), MGR Knowledge City, Cit CamPus, Taramani, Chennai 600 113, India.

E-mail address: pmuthumaths@gmail.com

S. Ponnusamy, Indian Statistical Institute (ISI), Chennai Centre, SEtS (Society for Electronic Transactions and Security), MGR Knowledge City, Cit Campus, Taramani, Chennai 600 113, India.

E-mail address: samy@isichennai.res.in, samy@iitm.ac.in 